\title{
Frontières
}

\section{L'Euthanasie en Belgique}

\section{Marie-Luce Delfosse}

Volume 24, numéro 1-2, automne 2011, printemps 2012

L’aide médicale à mourir

URI : https://id.erudit.org/iderudit/1013079ar

DOI : https://doi.org/10.7202/1013079ar

Aller au sommaire du numéro

Éditeur(s)

Université du Québec à Montréal

ISSN

1916-0976 (numérique)

Découvrir la revue

Citer ce document

Delfosse, M.-L. (2011). L’Euthanasie en Belgique. Frontières, 24(1-2), 9-10.

https://doi.org/10.7202/1013079ar

Ce document est protégé par la loi sur le droit d'auteur. L'utilisation des services d'Érudit (y compris la reproduction) est assujettie à sa politique d'utilisation que vous pouvez consulter en ligne.

https://apropos.erudit.org/fr/usagers/politique-dutilisation/ 


\section{L'EUTHANASIE EN BELGIQUE}

Marie-Luce Delfosse, Ph. D. codirectrice du numéro.

En 2002, la Belgique a adopté trois lois qui exercent une influence considérable sur l'éthique de la pratique médicale: la loi relative à l'euthanasie (28 mai), la loi relative aux soins palliatifs (14 juin) et la loi relative aux droits du patient (22 août) ${ }^{1}$. La première et la deuxième loi visent à assurer aux patients la possibilité de choisir la modalité de fin de vie qui correspond à leurs préférences, l'une en dépénalisant sous conditions l'acte d'euthanasie pratiqué par un médecin à la demande du patient, l'autre en posant le droit pour chacun de bénéficier de soins palliatifs et en prévoyant les dispositions pratiques qui permettent l'exercice de ce droit. La troisième loi, axée sur les droits individuels du patient, restructure la relation médecin-patient et vise à instaurer autant que possible celle-ci comme un partenariat entre personnes autonomes. Ces trois lois sont étroitement solidaires. Un sénateur l'a souligné : elles constituent un «triptyque » dont les différents éléments sont soutenus par le même esprit.

Ces lois furent adoptées après de nombreuses consultations et de très longues et larges auditions, suivies de débats nourris dans les assemblées parlementaires et les commissions. Furent entendus des représentants de l'Ordre des médecins, du Comité consultatif de bioéthique, d'associations de malades et de droits de l'homme, des médecins spécialistes et généralistes, des infirmiers, des pharmaciens, des juristes, un patient et un médecin hollandais.

Fait remarquable: les auditions furent le plus souvent publiques et diffusées par la télévision, en plus de leur relation, plus synthétique, dans les autres médias. Cette «publicité » demeure puisque la transcription intégrale des auditions publiques reste accessible sur le site du Sénat ${ }^{2}$ ainsi que dans les documents parlementaires.

L'ampleur de ces auditions montre que, confronté aux questions de la fin de vie, le législateur belge n'a pas voulu agir de façon seulement impérative, mais au contraire prendre appui sur l'expérience des acteurs de terrain et des experts. Ceux-ci avaient déjà été sollicités avant même ces auditions ainsi qu'en témoigne notamment le premier avis rendu en 1997 par le Comité consultatif de bioéthique de Belgique concernant l'opportunité d'un règlement légal de l'euthanasie ${ }^{3}$.

Toutes ces façons de procéder révèlent l'importance donnée à une démarche à partir de la base pour l'élaboration du texte de la loi.

En envisageant de légiférer sur la fin de vie, les Belges avaient pleinement conscience qu'ils s'engageaient à traiter une matière infiniment délicate. La préoccupation était de permettre à chacun, patient, médecin, soignant, de vivre cette étape de la vie d'une manière qui corresponde à ses convictions et à ses 
souhaits. C'est pourquoi l'euthanasie et les soins palliatifs ont fait l'objet de deux lois adoptées quasi simultanément de façon à offrir à chaque citoyen la possibilité de se tourner vers l'une ou l'autre orientation. La loi sur les droits du patient qui a suivi très rapidement confirme cette volonté d'assurer l'autonomie décisionnelle du patient, non comme un absolu en soi, mais dans le dialogue avec le médecin.

Les textes réunis dans le dossier sur l'euthanasie en Belgique qui est proposé aux lecteurs de Frontières visent à rendre compte des dispositions qui ont été adoptées en Belgique et de leurs incidences sur l'éthique et la pratique médicale ainsi que sur les mentalités. À partir de sa discipline propre, droit, médecine, philosophie, chaque auteur rend compte des enjeux qui affleurent dans sa réflexion non seulement à la lecture attentive du texte de la loi, mais aussi en référence à sa pratique en relation avec celle-ci. La jonction, dans chaque texte, d'une approche théorique et pratique permet ainsi de dépasser l'analyse du strict prescrit légal pour montrer les résonances de celui-ci et la dynamique de réflexion qu'il a ouverte au sein de la profession médicale, voire plus largement de la société.

Il importe d'aborder ces textes en ayant bien présente à l'esprit la définition que la loi belge donne de l'euthanasie: «acte, pratiqué par un tiers, qui met intentionnellement fin à la vie d'une personne à la demande de celle- $\mathrm{ci}^{4} »$. En effet, cette définition est décisive pour plusieurs raisons. D'abord, elle ouvre pour le patient un droit de demander l'euthanasie et non un droit à l'euthanasie. Ensuite, elle confie au seul patient la décision de formuler la demande: celle-ci n'est donc pas une initiative du médecin, mais du patient. Enfin, elle ouvre un espace relationnel entre le patient et le médecin : le patient formule sa demande, le médecin la reçoit et il lui revient alors d'évaluer si les conditions posées pour y répondre positivement sont respectées dans la situation particulière, tout en sachant qu'il peut faire valoir l'objection de conscience à condition de l'exprimer en temps utile; la décision prise finalement procède d'une conviction partagée par le médecin et le patient qui se forge au cours d'entretiens répétés. La définition spécifique que le législateur belge a adoptée situe donc la question dans un champ nettement délimité qui exclut d'autres distinctions et pratiques telles que l'euthanasie passive ou arrêt de traitement, l'euthanasie indirecte, notamment la sédation palliative ou terminale.

Jacqueline Herremans rend compte de la teneur juridique de la loi relative à l'euthanasie. Elle retrace d'abord le contexte qui a précédé l'élaboration de celle-ci : l'état du droit et de la déontologie médicale ainsi que les débats au sein de la société belge, notamment le rôle important joué par le Comité consultatif de bioéthique de Belgique. Elle relate ensuite les grandes étapes des débats parlementaires et l'avis rendu par le Conseil d'État exprimant la compatibilité du texte légal avec les dispositions de la Convention européenne de sauvegarde des droits de l'homme et des libertés fondamentales ainsi qu'avec le Pacte international relatif aux droits civils et politiques. Abordant la loi elle-même, elle en dégage les principes fondateurs et les diverses conditions posées, tant substantielles que de forme et de procédure.

Fernand Van Neste développe un aspect particulier de ces conditions de procédure: les travaux de la Commission fédérale de contrôle et d'application de la loi relative à l'euthanasie. Celle-ci a institué cette Commission pluridisciplinaire en la chargeant du suivi des actes d'euthanasie tels qu'ils se dégagent des documents d'enregistrement que les médecins, ayant pratiqué une euthanasie, sont tenus d'introduire auprès de cette Commission. Outre les modalités concrètes de contrôle mises en place par la loi et la Commission et leurs raisons d'être, le texte de Fernand Van Neste permet de donner un tableau d'ensemble de la situation en Belgique, de cerner les différences notables qui existent entre la partie néerlandophone et la partie francophone du pays, ainsi que les difficultés rencontrées et les questions qui demeurent ouvertes et relancent les débats. On perçoit ainsi très nettement que la loi n'a pas clos, mais au contraire ouvert une dynamique de réflexion au sein de la profession et de la société.

Trois médecins témoignent ensuite de leur expérience. Chacun d'eux exerce une activité médicale spécifique: Philippe Van Vlaenderen est généraliste; Gérard Marin l'est aussi et exerce en maisons de repos et de soins; Nadia Huart est oncologue et responsable d'une unité de soins palliatifs. Tous les trois ont reçu des demandes d'euthanasie qui les ont fortement interpellés. Ils nous font part des réflexions que ces demandes ont suscitées en eux, et de l'élaboration progressive de la réponse qui leur a semblé la plus juste. Plus précisément, ils récusent unanimement la polarisation souvent établie entre soins palliatifs et euthanasie, comme bonne et mauvaise prise en charge. De même, ils sont très attentifs à demeurer dans le cadre tracé par la loi à travers sa définition de l'euthanasie : ils refusent par conséquent d'assimiler l'euthanasie à des pratiques qui relèvent de la décision unilatérale du médecin, et qui ne constituent donc pas des réponses bien pesées à la demande réfléchie et répétée du patient. Pour eux, entendre une demande d'euthanasie et l'examiner au regard des possibilités existantes d'intervention thérapeutique et de soulagement de la douleur, accompagner le patient dans son cheminement constituent des devoirs professionnels exigeants, lourds mais à ne pas esquiver, et qui s'inscrivent dans le droit fil de leurs engagements en tant que médecins, en tant que croyants au service du respect de leurs patients.

En clôture de ce dossier, Marie-Luce Delfosse propose une réflexion philosophique qui prolonge les témoignages des trois médecins et s'inscrit dans le cheminement interdisciplinaire réalisé au sein d'un comité d'éthique hospitalière. Concernant les enjeux éthiques de la loi relative à l'euthanasie, elle montre qu'au-delà de l'affirmation de l'autonomie du patient, un autre enjeu plus fondamental se discerne, qui est sans doute paradoxal à première vue: le respect de l'intégrité mutuelle du patient et du médecin. Scruter la notion d'intégrité et particulièrement d'intégrité morale permet d'en montrer la teneur et la dimension dialogale. Cette analyse, soutenue par les approches de Paul Ricœur et d'Henri Ey, permet de proposer des repères éthiques aux médecins confrontés à des demandes d'euthanasie.

De ces textes sur l'euthanasie en Belgique se dégage une vision renouvelée de la mort. En effet, celle-ci constitue un moment que l'on veut généralement maintenir hors d'atteinte afin d'éviter des dérives. En donnant aux patients la possibilité de demander l'euthanasie, le législateur et tous ceux qui ont contribué à l'élaboration de la loi ont permis à la société belge de s'engager dans la voie d'une humanisation de ce moment final de la vie, ce que les soins palliatifs visent aussi d'une manière différente. Certes, la voie ouverte par la dépénalisation sous conditions de l'euthanasie est exigeante et demande beaucoup de vigilance. Cependant, à terme, elle apporte un apprivoisement et un respect de la mort qui devient alors, pleinement et au sens fort, l'étape ultime de la vie.

\section{Notes}

1. Moniteur belge, respectivement: 22 juin 2002, 26 octobre 2002, 6 octobre 2002 .

2. Voir sur le site $<$ http ://www.senate.be $>$ la rubrique «Quelques dossiers marquants ».

3. Voir sur le site <http://www.health.belgium.be/bioeth>, sous la rubrique «Avis » : avis $n^{\circ} 1$; voir aussi avis $n^{\circ} 9$.

4. Loi du 28 mai 2002 relative à l'euthanasie, art. 2. 